Als im Herbst 2000 ORI-HEX auf den Markt kam, gab es bereits viele antibakterielle Mundspüllösungen mit Chlorhexidindigluconat, dem nach Fluorid am häufigsten untersuchten Wirkstoff in der Zahnmedizin.

Mit ORI-HEX konnte die Firma ORIDIMA zur Markteinführung eine Rezeptur präsentieren, die erstmalig neben dem bewährten Chlorhexidin $(0,1 \%)$ zusätzlich Xylit enthielt. Xylit ist ein natürlicher Zuckeraustauschstoff, der aus der Rinde von Birken gewonnen wird, das Wachstum säurebildender Bakterien hemmt und damit das Wirkungsspektrum von Chlorhexidin ideal ergänzt.

Gleichrangig neben dieser Wirkkomponente sind im Zusammenhang mit Xylit aber auch die besonderen Süßungseigenschaften dieses natürlichen Zuckeraustauschstoffes hervorzuheben. Der strenge Eigengeschmack von Chlorhexidin konnte bei ORI-HEX dadurch deutlich reduziert werden. Ein wichtiger Aspekt zur Unterstützung der notwendigen Patienten-Compliance, vor allem bei Kindern und Jugendlichen.

Neben der Standardgröße 300 ml gibt es von ORI-HEX auch ein Zielspray $30 \mathrm{ml}$, mit dem Problemzonen im Mund- und Zahnbereich punktuell eingesprüht werden können. Aber auch für unterwegs, wenn Zahnpflege nicht möglich ist, hat sich ORI-HEX Spray sehr gut bewährt.

Anlässlich des zehnjährigen Jubiläums von ORI-HEX möchte sich ORIDIMA mit besonders günstigen Angeboten bei seinen treuen Verwendern bedanken. Und Interessenten möchten wir mit attraktiven Angeboten das Kennenlernen leicht machen.

Fragen Sie Ihren persönlichen Medizinprodukte-Berater vor Ort. Wir helfen Ihnen aber auch gerne weiter, wenn Sie uns anrufen, ein Fax oder eine E-Mail schicken.

入 Tel. 08542 - 89870-0

info@oridima.de

www.oridima.de

Komet/ Gebr. Brasseler

\title{
Schallspitze SFS110 ergänzt Winkelmodulationssystem
}

Das Angle Modulation System (Winkelmodulationssystem), das eine minimalinvasive horizontale Kammverbreiterung erlaubt, wird um eine wichtige Komponente erweitert: die Schallspitze SFS110. Das inzwischen eindrückliche Angebot an Komet-Schallspitzen bekommt damit ein neues, diamantiertes und konisches Familienmitglied, das punktuell bei den entscheidenden Arbeitsschritten einer Winkelmodulation zum Einsatz kommt. Mit ihr arbeitet der Operateur an der kortikalen Lamelle des zuvor crestal aufgetrennten Kieferkammes. Soll z.B. ein
Einzelimplantat inseriert werden, so schafft die SFS110 die vertikalen Ritzungen distal und mesial unter minimal-invasiven Bedingungen. Bei größeren horizontalen Kieferkammerweiterungen, wo mehrere Implantate nebeneinander eingebettet werden sollen, können mit der SFS110 gezielt Vertiefungen zur Mobilisierung gesetzt werden, damit anschließend mit Hilfe von Spread-Condense Schrauben und Modulatoren die bukkale Knochenlamelle geweitet werden kann. Wie alle Schallspitzen passt auch die SFS110 auf das SF1LM, das luftbetriebene und effizi- ente Schallhandstück von Komet. Damit stellt der Instrumentenhersteller erneut seinen Systemgedanken professionell unter Beweis.

入 Tel. 05261 - 701-700

info@brasseler.de

www.kometdental.de

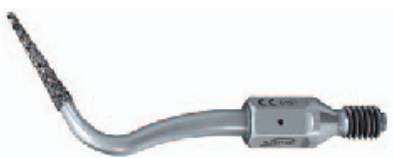

Baumgartner \& Rath GmbH

\section{Für ein strahlendes Lächeln - Praxisdigitalisierung ohne Lücken}

Kein Zahnarzt kommt heute an einer Praxisdigitalisierung vorbei. Der Umgang mit der Technik wird dabei immer selbstverständlicher, jedoch auch umfangreicher. Ging es früher lediglich darum, Karteikartenführung und Abrechnung in ein stimmiges EDV-System zu überführen, stellt die heutige Zeit ganz andere Anforderungen: die digitale Diagnostik revolutioniert die Praxis. Dadurch sind die Ansprüche an Hard-und Software gestiegen. Damit sich Arzt und/oder Zahnarzt im digitalen Dickicht zurecht finden können, brauchen sie einen Systemanbieter, der von Anfang an auf der Basis eines sicheren und umfassenden Konzeptes eine funktionierende EDV sowie alle nötigen und gewünschten Gerätekomponenten installiert. Und dies mit dem nötigen Branchen Know-how, zeit-und kostensparend. Die Baumgartner \& Rath GmbH hält für die perfekt ausgestattete Praxis ein umfassendes Sortiment an digitaler Dentaltechnik bereit. Einen Überblick kann man sich im unternehmenseigenen Showroom verschaffen: In einer voll funktionsfähigen Praxis sind alle Gerätekomponenten zu besichtigen und - zu testen, auch am Patienten. Highlights sind die neusten 2D-und 3D-Röntgenapparate u.a. des finnischen Herstellers Planmeca. Eine rundum digitalisierte Zahnarztpraxis ist heute Standard. Eine „lückenlose" Soft-und Hardwarelösung sollte es auch sein.

\section{ス Tel. 089 - 5420001}

info@baumgartner-rath.de www.baumgartner-rath.de www.hightech-dentaldepot.de

\section{BAUMGARTNER}

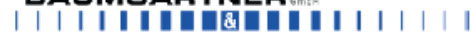

